\title{
Placebo is not a "sugar pill" but the result of stimulation of neural networks involved in central analgesia and in mood regulation
}

\author{
Maria Nicolodi ${ }^{1}$ \\ ${ }^{1}$ Foundation Prevention and Therapy of Primary Pain and Headache, Florence, Italy.
}

\begin{abstract}
Placebo was intended as a pill devoid of any active principle capable to influence or relieve so called psychosomatic diseases. More recently, it has been shown that placebo can be useful in surgical practice. In fact, sham operation can induce significant benefit. Emotions have been shown to play a pivotal role in placebo. Present observation concerns an attempt to investigate and therapeutically use scientific evidences in a placebo devoted to sufferers from chronic migraine pain. The present approach has its background in the fact that placebo analgesia has been shown to largely consist either in the activation both of analgesia pathways and of emotional systems as well as genetic and epigenetic expressions leading to specific behavior. In this perspective placebo has been used to empower a conventional therapy that was scored as unsatisfactory by the enrolled chronic migraine sufferers. Here proposed placebo seems to transform an ineffective drug therapy into an effective pain relieving approach
\end{abstract}

Keywords: Placebo; Placebo Analgesia; Endogenous Opiods; Non Opiodergic Systems: Environmental Conditions; chronic Migraine.

\section{Introduction}

What is placebo analgesia?

Placebo is often considered a "fake pill" as indicated in Randomized Controlled Trials - RCT- guidelines. In all cases, the "thing" named placebo has to influence networks controlling affective status-emotion ${ }^{[1-3]}$.

Endogenous opioids and non-opiodergic systems, namely dopamine and endocannabinoids, are involved in placebo analgesia. Similarly, GABA system is a limiting/controlling system involved in central analgesia and placebo analgesia ${ }^{[4]}$. All the mentioned neurotransmitters are well known to play a crucial role in depression, anxiety, rage, reward and pleasure and placebo effect amplitude ${ }^{[5-8]}$. Connections between emotion and placebo ${ }^{[8]}$ as well as epigenetic changes in neurotransmitters/receptors expression linked to placebo social experiences and emotions ${ }^{[9]}$ represented the ground to propose the present observations regarding "nurturing of social brain" that we propose as a placebo tool. Indeed, it has been shown that rodents that receive a maternal nurturing poor in grooming and liking, show an altered gene expression regarding dopamine and GABA neurochemical receptors. Environmental experiences might turn "on" or "off" the aforementioned receptors and transmitters through epigenetic pathway: that can lead to different behaviors also regarding pain.

\section{Materials and Methods}

Copyright (C) 2018 Maria Nicolodi.

doi: $10.18686 /$ jn.v7i1.135

This is an open-access article distributed under the terms of the Creative Commons Attribution Unported License

(http://creativecommons.org/licenses/by-nc/4.0/), which permits unrestricted use, distribution, and reproduction in any medium, provided the original work is properly cited. 
Our investigation started December 2015.

Enrolled subjects $\mathrm{n}=273$ (132 males, 141 females, mean age 39.2+ 3.2 SD) suffering from migraine chronic without aura - IHCD 3rd Beta Classification ${ }^{[10]}$.

Exclusion criteria were: psychiatric illness, ocular diseases, hearth diseases and arrhythmias, abnormal routine examines. Inclusion criteria were: refractoriness to conventional preventive therapies for chronic migraine, namely amitriptyline and topiramate ${ }^{[11]}$, absence of adverse induced by topiramate and/or amitriptyline.

Observation 1

Volunteers were divided in 2 matched groups:

Group 1= 66 males, 71 females; mean age 38.9+3.1 SD and Group B = 66 males, 70 females; mean age $38.8+2.9$ SD

The 2 group were compared to measure the homogeneity of the sample under observation regarding severity of pain experience (VAS 0-100), the rating of positive and negative affective state (PANAS) scales ${ }^{[12]}$. Main tool of Observation 1 was Affective Neuroscience Personality Scales ${ }^{[13]}$ that estimates primary processes of emotional traits of the patients. The latter measurement was propaedeutic to propose a powerful placebo that was to be employed in Observation 2.

Observation 2

All the 273 patients suffering from chronic migraine refractory to conventional treatment volunteered the observation. They were free to drop-out the observation in any moment. Migraine sufferers were randomly divided in Group A and Group B. Both the groups received a treatment based on outcomes of Observation 1. Thus, Group A (66 males, 71 females mean age $38.9+3.1 \mathrm{SD}$ ) and Group B (66 males, 70 females mean age $38.8+2.9 \mathrm{SD}$ ) were allocated to a social nurturing approach aimed to counteract fear-rage/despair.

Nurturing consisted in a guided meditation where the voices of a physician and a nurse are intertwined with music tracks with 430 Hertz frequency. The so structured sound tracks were to be heard at least 3 times/ $15-150$ min/daily.

Group A received topiramate $100 \mathrm{mg} /$ day/oral route. Patients included in Group A indicated topiramate as ineffective but devoid of adverse effects. Group B was administered amitriptyline $50 \mathrm{mag} / \mathrm{day} /$ oral route. In this group of patients the drug previously induced a benefit lower than $10 \%$ and evoked no adverse event. Escape medication was sumatriptan $100 \mathrm{mg} /$ orally. Plan:14 days run-in, 3 months treatment.

\section{Ethics Statement}

Thesubjects under observation volunteered to partecipate in Observation 1 and Observation 2 and gave their informed conset;

we respected the confidentiality and anonymity of our research respondents;

we ensured that our patients participated in our Observations voluntarily and that they could be free to drop out when if they desired;

we avoided to harm our participants in any moment;

we explained volunteers that the present observations were independent and impartial

\section{Results}

Observation 1 indicate that intractable chronic migraine without aura shows significant relationships $\quad p>0.0002$ (Odds Ratio and M-Anova) with systems thwart and arousal of rage/despair. The subjects in the sample group were homogeneous as regards pain severity mean VAS (0-100) and pain nonspecific affective state PANAS. Both in Observation 1 and Observation 2 there was no drop-out.

Observation 2 enlightened a great improvement ( $p>0.0001)$ in associating placebo administration to a conventional drug therapy previously indicated as non-effective. Indeed, after the treatment period with nurturing plus previously non effective drug, i.e. the approach described in Methods, Group A reported -26.9 + 1.8 SD hours/ pain p $>0.0001$ versus $-2.1+2.2 \mathrm{SD} ; \quad-48.0+5.3$ attacks severity $\mathrm{p}>0.0001$ versus $-3.6+2.4 \mathrm{SD}$ (VAS $0-100$ ) induced by non 
effective preventive therapy. Following the treatment period with nurturing approach and previously ineffective drug therapy, Group B reported a - 26.5 + 1.2 SD hours/pain p>0.0001 versus $-6.3+1.4 \mathrm{SD} ;-45.1+3.1 \mathrm{SD}$ attacks severity (VAS $0-100$ ) versus $-08+0.7 \mathrm{SD}$ induced by previous non effective conventional drug therapy.

\section{Discussion and Conclusions}

It could be inferred that placebo analgesia is powerful and involves emotional networks. It seems noteworthy that placebo analgesia can turn a previously ineffective pharmacological therapy into a pain ameliorating approach. It seems possible to conclude that the medical/nurse - patient interaction seems to have a great impact and value in achieving results in a pharmacological therapy. Indeed, the already known "positive care placebo effect" might largely be increased in case we specifically act on pathways regulating both emotions and analgesia.

\section{Conflict of Interest}

I declare that there is no conflict of interest.

\section{Funding}

I declare I did non receive any financial and non-financial support

\section{References}

1. Siegel A. The neurobiology of aggression and rage, CRC Press-Taylor \& Francis UK. 2004.

2. Panksepp J, Fuchs T, Iacobucci P. The basic neuroscience of emotional experiences in mammals: the case of subcortical fear circuitry and implications for clinical anxiety, Applied Animal Behav Sc. 2010; (5): 328-39

3. Nicolodi M, Torrini A. From nocebo effect to the hypothesis of nocebo comparison and psychometric tests as entry criteria in headache trials. Cephalalgia. 2009; 29(1): 39-40.

4. Zhuo M. Central inhibition and placebo analgesia Mol. Pain. 2005;(1):21-22.

5. Lutz P, Kieffer B. Opioid receptors: distinct roles in mood disorders, Trends Neurosci. 2012; 36(3): $195-206$.

6. Narvaes R, Martins De Almeida RM. Aggressive behaviour and three transmitters: dopamine, GABA, serotonin- a review of last 10 years, Psychology \& Neuroscience, 2014; 7(4): 601-607.

7. Wise RA, Rompre PP. Brain dopamine and reward, Annual Review of Psychology, 1989; (40): 191-225.

8. Flaten MA, Alasksen PM, Lybby P, et al. The relation of emotions to placebo responses, Philos Trans R B Biol Sci , 2011; 366 (1572):1818-27.

9. Champagne FA. Nurturing nature social experiences and brain, J. Endocrinology. 2009; 12 (10): 867-868.

10. The International Classification of Headache Disorders, 3rd edition (beta version). Headache Classification Committee of the International Headache Society (IHS). Comment in Cephalalgia 2013; 3(9):627-8.

11. Dodick DW, Freitag F, Banks J et al. Topiramate versus amitriptyline in migraine prevention: a 26-week, multicenter, randomized, double-blind, double-dummy, parallel-group non inferiority trial in adult migraineurs. Clin Ther . 2009; 31(3):542-59.

12. Watson D, Clark LA, Teleggen A. Development and validation of brief measures of positive and negative affects: the PANAS scales. J Personal Soc Psychol, 1998;(54):1063-1070.

13. Barrett FS, Robinson RW, Janata P. A brief form of the Affective Neuroscience Personality Scales, Psychol Assess. $2013 ; 25(3): 826-43$. 